\title{
POLARIS: a prospective, multicenter, noninterventional study assessing palbociclib in hormone receptor-positive advanced breast cancer
}

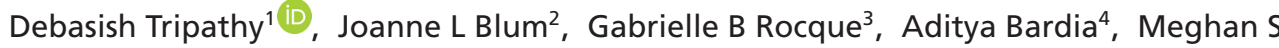 \\ Karuturi ${ }^{1}$, Joseph C Cappelleri ${ }^{5}$, Yuan Liu ${ }^{5}$, Zhe Zhang ${ }^{5}$, Keith L Davis*,6 \& Yao Wang ${ }^{5}$ \\ ${ }^{1}$ The University of Texas MD Anderson Cancer Center, Breast Medical Oncology, Unit 1354, 1515 Holcombe Blvd, Houston, TX \\ 77030, USA \\ ${ }^{2}$ Texas Oncology, 3410 Worth Street, Suite 400, Dallas, TX 75246, USA \\ ${ }^{3}$ The University of Alabama at Birmingham, 701 20th Street South, 1170 Administration Building, Birmingham, AL 35294, USA \\ ${ }^{4}$ Massachusetts General Hospital, 55 Fruit Street, LRH 304, Boston, MA 02114, USA \\ ${ }^{5}$ Pfizer Inc, 235 E. 42nd Street, New York, NY 10017, USA \\ ${ }^{6}$ RTI Health Solutions, 3040 East Cornwallis Road, Research Triangle Park, NC 27709, USA \\ *Author for correspondence: kldavis@rti.org
}

This report describes the rationale, purpose and design of the POLARIS study. POLARIS is an ongoing noninterventional, prospective, multicenter study. Female and male patients in the USA and Canada diagnosed with hormone receptor-positive/HER2-negative metastatic breast cancer were enrolled in the study and treated with the cyclin-dependent kinase 4/6 inhibitor palbociclib when hormone receptor-positive/HER2negative metastatic breast cancer was deemed to be indicated by their physician. The study will provide real-world data on palbociclib prescribing and treatment patterns in routine clinical practice, associated clinical outcomes, treatment sequencing in the advanced/metastatic setting, patient quality of life and geriatric-specific assessments. The tumor genomic landscape in relation to clinical outcomes will be explored. POLARIS will identify benefits and side effects of palbociclib across multiple lines of therapy and in discrete subsets of patients.

Clinical Trial Registration: NCT03280303 (ClinicalTrials.gov).

First draft submitted: 2 June 2020; Accepted for publication: 8 July 2020; Published online:

13 August 2020

Keywords: HR+/HER2- breast cancer $\bullet$ palbociclib $\bullet$ POLARIS • real-world evidence

Breast cancer, like other malignancies, is increasingly being treated with more precise and targeted therapies [1]. The first targeted therapy was applied over 100 years ago with the introduction of oophorectomy [2], preceding even the discovery of estrogens and androgens and ultimately refined with pharmacologic agents targeting estrogen production and binding to its receptor [3]. More recently, the HER2 oncogene emerged as an actionable therapeutic target through a variety of mechanisms using antibodies, kinase inhibitors and antibody-drug conjugates [4]. The most common subset of breast cancer, estrogen receptor positive or progesterone receptor positive (ER + and $\mathrm{PR}+$ ), HER2 negative (HER2-) breast cancer, has historically been treated primarily with endocrine therapy. The rapid development of targeted agents that significantly improve the activity of endocrine therapies represents a welcome advance, but has left many questions regarding the optimal sequence to use over the course of the disease, the methods to better personalize treatments based on clinical and biological factors and the impact of these treatments on health-related quality of life (HRQoL). Here we summarize the rationale and methodology of a prospective registry designed to shed light on these questions in a real-world setting.

First-line treatment in postmenopausal patients with hormone receptor positive (HR+)/HER2- advanced or metastatic breast cancer $(\mathrm{MBC})$ includes endocrine therapies such as letrozole, anastrozole, exemestane and tamoxifen, with progression-free survival (PFS) ranging from 5 to 16 months [5-8]. These treatment options are

Future Medicine 


\begin{tabular}{|c|c|c|c|c|c|c|}
\hline \multirow[t]{2}{*}{ Characteristics } & \multicolumn{2}{|c|}{ PALOMA-1 } & \multicolumn{2}{|c|}{ PALOMA-2 } & \multicolumn{2}{|c|}{ PALOMA-3 } \\
\hline & $\begin{array}{l}\text { Palbociclib + } \\
\text { letrozole }(n=84)\end{array}$ & $\begin{array}{l}\text { Letrozole } \\
(n=81)\end{array}$ & $\begin{array}{l}\text { Palbociclib + } \\
\text { letrozole }(n=444)\end{array}$ & $\begin{array}{l}\text { Letrozole + placebo } \\
(n=222)\end{array}$ & $\begin{array}{l}\text { Palbociclib + } \\
\text { fulvestrant } \\
(n=347)\end{array}$ & $\begin{array}{l}\text { Fulvestrant }+ \\
\text { placebo }(n=174)\end{array}$ \\
\hline \multicolumn{7}{|c|}{ Progression-free survival } \\
\hline $\begin{array}{l}\text { - Median follow-up, } \\
\text { months }\end{array}$ & 29.6 & 27.9 & 37.6 & 37.3 & 8.9 & 8.9 \\
\hline $\begin{array}{l}\text { - Median PFS } \\
(95 \% \mathrm{Cl}) \text {, months }\end{array}$ & $\begin{array}{l}20.2 \\
(13.8-27.5)\end{array}$ & $\begin{array}{l}10.2 \\
(5.7-12.6)\end{array}$ & $\begin{array}{l}27.6 \\
(22.4-30.3)\end{array}$ & $\begin{array}{l}14.5 \\
(12.3-17.1)\end{array}$ & $\begin{array}{l}9.5 \\
(9.2-11.0)\end{array}$ & $\begin{array}{l}4.6 \\
(3.5-5.6)\end{array}$ \\
\hline \multicolumn{7}{|l|}{ Overall survival } \\
\hline $\begin{array}{l}\text { - Median follow-up, } \\
\text { months }\end{array}$ & - & & - & & 44.8 & 44.8 \\
\hline $\begin{array}{l}\text { - Median OS } \\
(95 \% \mathrm{Cl}) \text {, months }\end{array}$ & - & & - & & $\begin{array}{l}34.9 \\
(28.8-40.0)\end{array}$ & $\begin{array}{l}28.0 \\
(23.6-34.6)\end{array}$ \\
\hline
\end{tabular}

recommended by the European Society for Medical Oncology and National Comprehensive Cancer Network guidelines as preferred first-line options in patients who are antiestrogen naive or who are more than 2 years from previous antiestrogen therapy and do not have extensive visceral involvement [5,9]. Aromatase inhibitor or tamoxifen monotherapy has shown limited clinical benefit $[10,11]$. The selective estrogen receptor degrader, fulvestrant, has modest activity in this population beyond first line $[12,13]$.

Despite endocrine therapies being a mainstay of first-line treatment, some patients with HR+/HER2- MBC have de novo resistance to hormone-targeted agents and most other cases develop acquired resistance and eventually progressive disease [14,15]. Primary resistance is defined by the 2018 European Society for Medical Oncology guidelines as development of metastatic disease within 2 years of adjuvant treatment, or within 6 months of treatment for metastatic disease [16]. Secondary resistance is defined as the development of locally advanced or metastatic disease within 12 months of discontinuation of endocrine therapy; in the metastatic setting, secondary resistance develops after 6 months [16]. Initial therapy for these patients with visceral crisis often involves chemotherapy, which has significant toxicities. The development of effective therapies that can address resistance to endocrine therapy in these patients is therefore of clinical importance. In vitro evidence suggests that HR+ breast cancers that have developed resistance to prior endocrine therapy continue to rely on pathways that converge on CDKs to bypass a cell cycle checkpoint and promote proliferation [17-20]. As such, CDK inhibition has become an attractive target for novel therapies. Palbociclib is an orally bioavailable small-molecule inhibitor of CDK4 and CDK6 with a high level of selectivity for these targets over other CDKs [21]. Based on PFS results from the PALOMA clinical trials, palbociclib became the first CDK4/ 6 inhibitor approved by the US FDA for the treatment of HR+/HER2- MBC when used in combination with endocrine therapy. Palbociclib initially received accelerated approval for use in combination with letrozole as first-line therapy for postmenopausal women with estrogen receptor-positive/HER2- MBC based on results from the Phase II PALOMA-1 clinical trial. Subsequently, palbociclib was approved in combination with fulvestrant, in patients with disease progression following prior endocrine therapy, regardless of menopausal status, based on results from the Phase III PALOMA-3 trial. This was followed by expanded use in combination with an aromatase inhibitor as initial endocrine-based therapy based on results from the Phase III PALOMA-2 trial. Table 1 summarizes PFS results with palbociclib plus letrozole from PALOMA-1 [14] and PALOMA-2 [15], as well as the PFS [22] and overall survival (OS) results [23] with palbociclib plus fulvestrant from PALOMA-3. Of note, a recent report found that median PFS was similar in a real-world cohort of women with HR+/HER2- advanced breast cancer who received letrozole monotherapy and in the placebo-letrozole arm of PALOMA-2 (18.4 and 16.6 months, respectively) [24], suggesting that a similar treatment effect with palbociclib combination therapy may be observed in the real-world setting, with broader generalizability with the use of real-world assessments of progression and tumor response.

Outside the PALOMA trials, palbociclib plus endocrine therapy has also been evaluated against chemotherapy (rather than against placebo plus endocrine therapy). In a recent report from a multicenter, open-label, Phase II trial of palbociclib plus exemestane (with ovarian suppression) versus capecitabine chemotherapy in premenopausal patients with HR+/HER2- MBC who relapsed or progressed on previous tamoxifen therapy, over a median 
follow-up of 17 months, median PFS was 20.1 months (95\% CI: 14.2-21.8) in the palbociclib plus endocrine therapy group versus 14.4 months (12.1-17.0) in the capecitabine group [25]. Findings from this study provide further evidence regarding the effectiveness of palbociclib in other treatment scenarios that could not be examined in the PALOMA studies.

CDK inhibitors, such as palbociclib and more recently ribociclib [26-28] and abemaciclib [29,30], represent an important advance in the treatment paradigm for HR+/HER2- MBC. However, HR+ breast cancers are biologically heterogeneous. Although CDK inhibitor trial results are promising, not all patients will respond to treatment, and many patients have varying susceptibility to certain mechanism-based toxicities such as neutropenia, leukopenia, anemia and thrombocytopenia [15]. As a result, there is a need to better understand the spectrum of clinical responses to CDK inhibition in HR+ breast cancer, management patterns in terms of dose adjustments or discontinuation and patients' experiences through patient-reported outcomes (PROs).

Biomarker analysis of the PALOMA-3 trial revealed that CDK4, CDK6, cyclin D1 and RB1 expression levels were not significantly associated with the benefit of palbociclib combination treatment, whereas high CCNE1 levels were associated with lower palbociclib benefit [31]. However, analyses from PALOMA-2 did not identify any biomarkers associated with clinical benefit from palbociclib combination treatment [32]. A better understanding of biomarkers that can predict which patients will potentially benefit from treatment with CDK inhibitors may also be valuable.

As the shifting treatment landscape makes it difficult to conduct randomized trials of all potential therapies and their combinations, prospective observational studies in routine practice settings (that include less-selective populations outside the tightly controlled environs of clinical trials) present an increasingly important method to obtain such data on CDK inhibitors in patients with HR+/HER2- MBC [33]. In this report, we introduce the POLARIS study, an ongoing noninterventional, prospective, multicenter study enrolling female and male patients in the USA and Canada diagnosed with HR+/HER2- MBC and treated with the CDK4/6 inhibitor palbociclib when deemed indicated by the attending physician. POLARIS will provide data from real-world settings on palbociclib prescribing and treatment patterns in routine clinical practice, associated clinical outcomes, treatment sequencing in the advanced/metastatic setting, patient quality of life and geriatric-specific assessments. An exploratory aim of POLARIS is to assess biomarkers' underlying resistance or response mechanisms to palbociclib treatment. Due to stringent clinical trial eligibility criteria and limited time of follow-up on study, additional tools (e.g., observational studies similar to POLARIS) are needed to identify projected benefits and side effects over multiple lines of therapy and in discrete subsets of patients, not only to improve routine clinical care and shared decision-making with patients, but also to better inform trial designs and sample size requirements going forward.

POLARIS is preceded by other breast cancer registries collecting data from real-world settings, including ATHENA [34], RegistHER [35-38], SystHERS and MedSIR (ClinicalTrials.gov: NCT03819010). Additionally, a standalone study collecting HRQoL data from patients with HER2+ early or metastatic breast cancer treated with trastuzumab or chemotherapy in routine practice has been published recently [39]. These registries and observational studies comport with the current growing interest in and use of real-world data sources in breast cancer research, particularly those linking electronic health records on treatment and outcomes to prospective cancer registries, to address clinically relevant questions that may not be answered in clinical trials [40]. POLARIS is distinct from the previously noted registries in that it has a broader collection of PRO data; includes longitudinal biomarker/genomics data and geriatric assessments; features planned subgroup analyses (e.g., geriatric patients); was designed a priori to be drug-specific (palbociclib), although noninterventional; and focuses solely on HR+/HER2- disease. POLARIS will therefore address several important knowledge gaps with palbociclib in the real-world management of HR+/HER2MBC disease. In the following sections, we explain the design and aims of POLARIS and discuss the potential applications and needs addressed by the data that will be collected.

\section{Construction \& content}

\section{Study design}

POLARIS is a prospective, noninterventional, multicenter study being conducted in the USA and Canada $(\mathrm{Cl}$ inicalTrials.gov: NCT03280303). A target of 1500 subjects will be enrolled across approximately 100 sites in the USA and ten sites in Canada. Participation in the study is not intended to change the routine treatment patients receive as determined by their prescribing physicians; all therapeutic decisions, as well as the type and timing of disease monitoring, are at the discretion of the treating physician and patient. Assessments include a review of patients' medical records, patient quality of life questionnaires (European Organisation for Research and 
Treatment of Cancer Quality of Life Questionnaire-Core 30 [EORTC QLQ-C30], G8 Screening Tool, Activities of Daily Living $[\mathrm{ADL}])$, site questionnaires, physician $\mathrm{MBC}$ treatment selection survey and biomarker assessments. Analysis of baseline and serial blood samples (circulating tumor DNA [ctDNA]) for markers of palbociclib response/resistance will be performed on consenting patients.

\section{Eligibility criteria \\ Inclusion criteria}

Patients meeting all of the following inclusion criteria are eligible for enrollment into the study:

1. Age $\geq 18$ years;

2. Diagnosis of invasive breast cancer with evidence of metastatic or advanced disease not amenable to treatment with curative intent;

3. Documented HR+ (estrogen receptor-positive and/or progesterone receptor-positive) tumor based on local standards;

4. Documented HER2- tumor based on local standards;

5. Physician determination that treatment with palbociclib is indicated;

6. Signed and dated informed consent documentation;

7. Willingness and ability, in the opinion of the investigator, to comply with regular clinic visits as per standardof-care practice at the site.

\section{Exclusion criteria}

Patients meeting any of the following criteria are excluded from the study:

1. Life expectancy of less than 3 months at the time of MBC diagnosis per the investigator's judgment;

2. Participation in any interventional clinical trial that includes investigational or approved drugs at the time of enrollment (patients participating in other investigator-initiated research or noninterventional studies can be included as long as their standard of care is not altered by the study);

3. On active treatment for malignancies other than $\mathrm{MBC}$ at the time of enrollment;

4. Inability to understand the nature of the study and/or unwillingness to sign an informed consent.

\section{Recruitment plan}

Patients with HR+/HER2- MBC in the USA and Canada will be enrolled from a geographically representative population as well as from diverse treatment settings (e.g., academic and community sites). Patients with MBC whose palbociclib treatment decision has been made by their treating physician and who meet the eligibility criteria will be invited to participate in the study. Eligibility will be assessed at the date of the enrollment visit (i.e., baseline/first standard of care visit).

\section{Study variables}

The following variables will be collected and assessed: patient demographics; study site characteristics; patients' general medical history and baseline concomitant medications; breast cancer diagnosis and recurrence history; metastatic disease status; cancer treatment selections in the advanced/metastatic setting; disease status and characteristics; Eastern Cooperative Oncology Group (ECOG) performance status; palbociclib and endocrine therapy dosing and treatment management; routine clinical efficacy assessment (including overall clinical response as assessed by the treating physician and survival); physician MBC treatment selection survey (including reasons for prescribing palbociclib); adverse events; quality of life; geriatric screening tool and questionnaire; and selected biomarkers. Data will be collected from the treating physicians' routine clinical assessments per their local standard of care.

Patients will be asked to complete the EORTC QLQ-C30 at enrollment (baseline) [41,42] and monthly for the first 3 months of treatment with palbociclib, then every 3 months until the end of treatment with palbociclib. The G8 Geriatric Screening Tool and ADL questionnaire [43,44] will be completed for patients aged $\geq 70$ years at time of study enrollment. The treating physician or appropriately delegated site staff will complete the G8 Screening Tool and will complete the patient ADL questionnaire in conjunction with the appropriately delegated site staff as needed. Similar to the EORTC QLQ-C30, the G8 Geriatric Screening Tool and ADL questionnaire will be completed monthly for the first 3 months of treatment with palbociclib and then every 3 months throughout 


\begin{tabular}{|c|c|c|c|}
\hline Enrollment & SOC visit 1 & \begin{tabular}{|c|}
$\begin{array}{c}\text { SOC visit } 2 \text { through SOC visit } \mathrm{N} \\
\text { (i.e., end of palbociclib } \\
\text { treatment) }\end{array}$ \\
\end{tabular} & Follow-up through end of study \\
\hline Data collection elements and entry ${ }^{\dagger}$ & (Baseline) & (During palbociclib treatment) & (After palbociclib treatment) \\
\hline Informed consent and inclusion/exclusion criteria & $\bullet$ & & \\
\hline $\begin{array}{l}\text { Patient demographics, study site characteristics, } \\
\text { medical history, baseline concomitant medications, } \\
\text { baseline metastatic disease status }\end{array}$ & $\bullet$ & & \\
\hline Breast cancer diagnosis and recurrence history & $\bullet$ & & \\
\hline Breast cancer treatment $\$$ & $\bullet$ & - & $\bullet$ \\
\hline Physician MBC treatment selection survey" & $\bullet$ & $\bullet$ & $\bullet$ \\
\hline Performance status (ECOG) & $\bullet$ & $\bullet$ & \\
\hline Clinical assessments $\#$ & $\bullet$ & $\bullet$ & $\bullet$ \\
\hline Biomarker blood sample ${ }^{\dagger \dagger}$ & $\bullet$ & $\bullet$ & \\
\hline Laboratory: CBC & $\bullet$ & $\bullet$ & \\
\hline Adverse events and serious adverse events $\$ \S$ & $\bullet$ & $\bullet$ & \\
\hline EORTC QLQ-C30 भा & $\bullet$ & $\bullet$ & \\
\hline G8 screening tool\#\# & $\bullet$ & $\bullet$ & \\
\hline ADL: activities of daily living & $\bullet$ & $\bullet$ & \\
\hline
\end{tabular}

Figure 1. Data entry schedule.

${ }^{\dagger}$ All data will be collected using an electronic data capture system through direct data entry by site clinical and research staff.

¥Follow-up data will be collected over the course of any SOC visits occurring after completion of palbociclib treatment.

$\S$ Breast cancer treatment includes surgery, radiation, systemic chemotherapy, hormone therapy and/or targeted therapy, in both neoadjuvant/adjuvant and metastatic disease settings, before, during and after palbociclib treatment; supportive care is also collected in the metastatic setting while on this study.

I Treating physicians will complete an MBC treatment selection survey to capture the reason(s) for treatment choice at start of palbociclib therapy and at the start of subsequent therapies.

\#Clinical assessments are based on overall clinical response as judged by the treating physician, at intervals per SOC schedule: imaging assessment, clinical progression and clinical biomarkers.

${ }^{\dagger}$ Biomarker blood sample, for patients providing additional consent: two tubes of blood (10 ml) will be collected at baseline (before start of palbociclib) and two tubes of blood $(10 \mathrm{ml})$ will be collected at end of palbociclib treatment. In between these points, one tube of blood $(10 \mathrm{ml})$ will be collected at day 1 of every cycle for the first 6 months after enrollment, then one tube of blood (10 ml) will be collected at day 1 of every three cycles per SOC until the end of treatment with palbociclib.

$\$$ $¥ B C$ will be captured at baseline and during palbociclib treatment.

$\S$ AE/SAE reporting begins at the time of the patient's first dose of palbociclib or the time of the patient's informed consent if already exposed to palbociclib and up to 28 calendar days following the last administration of palbociclib.

IIEORTC QLQ-C30 will be collected at baseline (before starting palbociclib) and then monthly for the first 3 months of treatment with palbociclib, then every 3 months until the end of treatment with palbociclib.

\#\#8 Screening Tool and ADL will be completed only for patients $\geq 70$ years old at time of study enrollment. G8 and ADL will be collected at baseline (before starting palbociclib), then monthly for the first 3 months of treatment with palbociclib, then every 3 months until the end of treatment with palbociclib.

ADL: Activities of daily living; AE: Adverse event; CBC: Complete blood count; ECOG: Eastern Cooperative Oncology Group; EORTC QLQ-C30: European Organisation for Research and Treatment of Cancer Quality of Life Questionnaire-Core 30; MBC: Metastatic breast cancer; SAE: Serious adverse event; SOC: Standard of care.

treatment with palbociclib. A subset of patients will be asked to provide optional blood samples (drawn at the time of standard-of-care laboratory samples) at baseline, during palbociclib treatment and at the end of palbociclib treatment for biomarker (including ctDNA) analysis. The study variables and data entry schedule are further described in Figure 1.

\section{ctDNA analyses}

Blood samples will be collected from patients who consent for ctDNA analysis at baseline (before palbociclib treatment), every cycle for the first 6 months, every three cycles thereafter and at the end of treatment. The ctDNA analysis of blood samples will be conducted using the Guardant360 platform (Guardant Health, Inc., CA, 
USA). Collection of longitudinal (baseline, on-treatment and at disease progression) samples will allow the use of ctDNA presence and/or absence during early treatment to predict palbociclib plus endocrine therapy responses and to explore potential intrinsic and acquired resistance mechanisms. It will also contribute to understanding the occurrences of gene mutations, fusions or amplifications in candidate genes, such as FGFR1-3, RB1 and GATA3, that may be involved in cell cycle control, cell growth, hormone regulation, apoptosis pathways, oncogenesis or tumor suppression. The baseline and change from baseline in ctDNA fraction and in mutation profiles during treatment will be evaluated for their potential predictability of treatment outcome. Dynamic ctDNA mutation profiles over the course of treatment will be analyzed to explore potential gain-of-function or loss-of-function mutations associated with response, resistance and disease progression.

\section{Statistical analyses \\ General considerations}

All patient characteristics and pathologic, treatment and genomic variables will be summarized descriptively through the tabular and graphical display of mean values, medians, ranges and standard deviations of continuous variables of interest and proportions and frequency distributions for categorical variables. Inferential analyses, coupled with descriptive statistics, will be considered to examine the relationship among variables such as baseline demographics, clinical characteristics, dose modifications, ECOG performance status and adverse events.

\section{Geriatric \& quality-of-life assessments}

Frailty and functional status will be assessed with the G8 and ADL screening tools, respectively. G8 and ADL scores will be captured at study visits and, in one analysis, their scores will be stratified by severity (G8: $\leq 14=\mathrm{im}$ paired, $>14=$ normal; ADL: $\leq 5=$ impaired, $>5=$ normal). Associations between treatment outcomes (ECOG, dose modifications, adverse events) and G8 and ADL scores at key time points (e.g., baseline and 6 months) will be analyzed with descriptive statistics and the Fisher exact test.

For the three quality-of-life outcomes (G8, ADL and EORTC QLQ-30), a paired $t$-test will be performed to examine mean change in scores from baseline to a postbaseline visit; a two-sample $t$-test or analysis of variance will be performed to examine means between groups at a particular visit (e.g., ECOG $=0$ vs ECOG $>0$ ). To interpret functional and symptom subscales/items on the EORTC QLQ-C30 scores in this population, they will be compared with their corresponding general population norm scores in the USA and Canada [45]. Although these quality-of-life tools may reflect the adverse effects caused by loss of estrogen activity due to the endocrine therapy, they will be useful to measure any additional changes in quality of life that may be caused by palbociclib treatment.

\section{Real-world clinical outcomes}

Real-world overall clinical tumor response (complete response, partial response, stable disease or progressive disease) as assessed by the treating physician will be summarized. A real-world best overall response rate will be estimated as the proportion of patients with real-world best overall response of complete response or partial response. The real-world clinical benefit rate, defined as the proportion of patients with complete response, partial response or stable disease at 24 weeks, will also be reported. The corresponding 95\% CI will be provided. Associations between levels of a subgroup or other covariates and real-world tumor response outcomes may be evaluated using chi-square tests and logistical regression models.

Real-world time-to-event outcomes (e.g., PFS, OS) will be described using the Kaplan-Meier method. Comparisons of survival distributions between groups will be made using a log-rank test. Subgroup analyses will be performed based on demographic and clinical factors (e.g., ECOG performance status, line of therapy, menopause status and age groups).

\section{Biomarkers}

Gene-level mutations/copy number changes, mutational profiles and changes in mutation ctDNA fractions over treatment from baseline will be summarized using descriptive statistics. For the potential predictive ability of the treatment effect, baseline and change in ctDNA mutation will be correlated with real-world clinical outcomes (e.g., real-world response, PFS and OS) using logistic regression and Cox proportional hazards models. Association of longitudinal biomarker trajectory over the course of treatment with real-world time-to-event outcomes will be explored. 


\section{Ethical considerations}

The study will be conducted in accordance with local legal and regulatory requirements to ensure protection of patients' personal data, including collection of patients' informed consent. As previously noted, separate consent is required for biospecimen acquisition for biomarker analyses. With these provisions, the study has been reviewed and approved by applicable local institutional review boards.

\section{Governance structure}

The POLARIS steering committee is the primary group responsible for the scientific integrity of the study. The purpose of the POLARIS steering committee is to provide objective scientific and medical input into the oversight, analysis and interpretation of data for the study. Specific responsibilities of the steering committee are: providing expertise and guidance on the design of the protocol and protocol amendments to achieve the study objectives; ensuring the scientific quality and integrity of the study; providing input for the statistical analysis plan; and reviewing and approving the publication of study results. The steering committee comprises six academic and community oncologists and one oncologist employed by the study sponsor; the steering committee chair is appointed by the study sponsor. The steering committee meets annually (at minimum) or more frequently as needed and operates under an approved charter, which specifies the roles and responsibilities of the committee members. The steering committee charter also describes how decisions will be made, how study priorities will be set and how plans will be executed.

\section{Methodologic limitations}

The POLARIS study will be subject to several of the methodologic limitations inherent to any noninterventional real-world study. These studies have the potential for missing, inaccurate or incomplete data. Furthermore, as a result of the observational design, patient selection and the diagnostic or monitoring procedures used are those applied per the usual treatment paradigm of the treating physician rather than being dictated by a protocol. Tumor assessments, for example, may be made on a less (or more) frequent basis in real-world practice than the typically mandated fixed schedule of an interventional trial protocol, and response is determined by the physician based on source evidence of clinical judgment, imaging, biopsies and/or biomarkers rather than objective Response Evaluation Criteria in Solid Tumors (RECIST) criteria. Thus, estimates of time to progression and PFS may not be directly comparable with those obtained from trial data. Heterogeneous, less-selective patient populations also increase the difficulty of interpreting outcomes data. Hence, this study is not intended to confirm causality between treatments and outcomes, but rather to provide complementary data to reported trial results.

\section{Utility \& discussion}

Although the treatment landscape for patients with HR+/HER2- MBC has evolved substantially in recent years, particularly with the approval and use of CDK4/6 inhibitors, there remains a surprising lack of real-world data on the natural history, treatment selections and outcomes of these patients following the advent of these new targeted therapies. POLARIS is designed to address these information gaps in the context of palbociclib treatment, with the inclusion of PROs, geriatric assessments and biospecimen collection intended to provide further insights on important aspects of treatment (i.e., disease evolution, sequential therapies, quality-of-life experiences and genomic correlates of treatment response) for which real-world data are lacking.

The inclusion of geriatric assessments in particular may address one initiative put forth in recent years by the American Society of Clinical Oncology (ASCO) to improve the evidence base for treating older adults with cancer [46]. Partially owing to underrepresentation of geriatric populations in cancer research and treatment [47-50], evidence on treatment outcomes and patient experiences with treatment (including quality of life) is sparse for geriatric adults, despite this population incurring the majority of cancer diagnoses and deaths and also representing the majority of cancer survivors. In 2015 a committee convened by ASCO made five recommendations to improve evidence generation in this population. One of these was to "leverage research designs and infrastructure for generating evidence on older adults with cancer." As concluded by the ASCO committee, one of the available designs that should be considered as a means to address this recommendation is the prospective observational cohort study, such as POLARIS. Subsequent to these deliberations, ASCO in combination with the FDA later cosponsored a follow-up workshop to further expand the evidence base in geriatric oncology. Similar to the previous committee recommendations, one key action item identified by the subsequent workshop was to expand the use of real-world data in research on older adults with cancer [51]. 
It is widely acknowledged that HRQoL in breast cancer survivors is compromised in different functional domains and that these areas need attention [52]. Furthermore, an earlier literature review also reported that, compared with younger patients, elderly patients with cancer tend to perceive HRQoL as more important than survival gains [53]. The broader capture of PRO data in POLARIS compared with some previous registries may help further address current knowledge gaps on HRQoL in patients (and in particular, older patients) with HR+/HER2- breast cancer.

The importance of collecting real-world data on CDK4/6 inhibitor treatment is increasingly acknowledged, and trials to collect these data are underway. A similar trial to POLARIS is in progress for ribociclib (RIBANNA), which will enroll approximately 3020 patients in Germany [54]. RIBANNA is a noninterventional study that will collect data on efficacy, safety, duration of therapy and quality of life. Together, these two trials will provide necessary data on the real-world use of CDK4/6 inhibitors [54].

\section{Conclusion}

POLARIS is positioned to collect extensive and granular follow-up data on patients with HR+/HER2- MBC who have received palbociclib, thereby elucidating the longer-term use, sequencing and effectiveness of CDK $4 / 6$ inhibitors in this patient population. $\mathrm{HR}+/ \mathrm{HER} 2-\mathrm{MBC}$ has a long natural history; therefore, longer-term followup over multiple courses of therapy, as expected from POLARIS, is essential. Other population-based sources of data (e.g., SEER-Medicare) could also complement registry data like those to be collected from POLARIS. Although the number of patients is a strength of this approach, weaknesses include the lower level of data complexity and accuracy. Finally, longitudinal biomarker data should increasingly be coupled with outcomes data to discern the biology associated with treatment response and resistance and to fuel discovery. As the utility and cost-effectiveness of such assays are increasingly becoming more integrated into routine care, there is a greater opportunity to collect these data and examine their association with outcomes for potential use in posttreatment surveillance of patients with cancer to discover new therapeutic targets.

\section{Executive summary}

\section{Background}

- CDK 4/6 inhibitors have been incorporated into the treatment paradigm for hormone receptor-positive (HR+)/HER2-negative (HER2-) metastatic breast cancer, but patients' response to CDK4/6 inhibitors may vary.

- The efficacy of palbociclib plus endocrine therapy has been demonstrated in the PALOMA clinical trial program; findings from the POLARIS study will provide further evidence regarding the effectiveness of palbociclib in other treatment scenarios that could not be examined in the PALOMA studies.

- POLARIS will provide real-world data on palbociclib prescribing and treatment patterns in routine clinical practice, associated clinical outcomes, treatment sequencing in the advanced/metastatic setting, patient quality of life and geriatric-specific assessments, as well as explore prognostic and predictive features of tumor genomics.

Construction \& content

- POLARIS is a prospective, noninterventional, multicenter study with a target enrollment of 1500 patients across approximately 100 sites in the USA and ten sites in Canada.

- Key inclusion criteria are patients aged $\geq 18$ years with HR+/HER2- advanced or metastatic breast cancer where the physician has determined that palbociclib therapy is indicated.

- Data, including patient demographics and clinical characteristics, palbociclib and endocrine therapy dosing and treatment management and routine clinical efficacy assessments, will be collected from the treating physicians' routine clinical assessments. Baseline and longitudinal circulating tumor DNA will be obtained and analyzed.

Utility \& discussion

- There is a lack of real-world data on the natural history, treatment selections and outcomes of patients treated with a CDK4/6 inhibitor.

- POLARIS addresses these information gaps in the context of palbociclib treatment.

Conclusion

- POLARIS will collect data on patients with HR+/HER2- metastatic breast cancer who have received treatment with palbociclib, thereby elucidating the longer-term use, sequencing and effectiveness of CDK4/6 inhibitors in this patient population. 


\section{Author contributions}

D Tripathy, J Blum, G Rocque, A Bardia, M Karuturi, J Cappelleri, Y Liu, Z Zhang and Y Wang participated in the conception and design of the POLARIS study and provided critical review, revision and approval of article drafts. K Davis drafted the article and subsequent revisions and provided critical review and approval of all article drafts.

\section{Financial and competing interests disclosure}

This study is sponsored by Pfizer Inc. D Tripathy, J Blum, G Rocque, A Bardia and M Karuturi received remuneration from Pfizer Inc for their services as members of the steering committee for POLARIS. J Cappelleri, Y Liu, Z Zhang and Y Wang are employees of and own stock in Pfizer Inc. K Davis is an employee of RTI Health Solutions, which received financial support from CPI Global in connection with the development of this manuscript. CPI Global received financial support from Pfizer Inc in connection with the conduct of this study. The authors have no other relevant affiliations or financial involvement with any organization or entity with a financial interest in or financial conflict with the subject matter or materials discussed in the manuscript apart from those disclosed.

The support provided by Anny Wu of ICON plc (PA, USA) and funded by Pfizer Inc consisted solely of manuscript formatting; no contribution was made to editorial content.

\section{Ethics conduct of research}

The study will be conducted in accordance with local legal and regulatory requirements to ensure protection of patients' personal data, including collection of patient informed consent. Separate consent is required for biospecimen acquisition for biomarker analyses. With these provisions, the study has been reviewed and approved by applicable local institutional review boards.

\section{Open access}

This work is licensed under the Attribution-NonCommercial-NoDerivatives 4.0 Unported License. To view a copy of this license, visit http://creativecommons.org/licenses/by-nc-nd/4.0/

\section{References}

Papers of special note have been highlighted as: $\bullet$ of interest; $\bullet \bullet$ of considerable interest

1. Waks AG, Winer EP. Breast cancer treatment: a review. JAMA 321(3), 288-300 (2019).

2. Beatson GT. On the treatment of inoperable cases of carcinoma of the mamma: suggestions for a new method of treatment, with illustrative cases. Trans. Med. Chir. Soc. Edinb. 15, 153-179 (1896).

3. Turner NC, Neven P, Loibl S, Andre F. Advances in the treatment of advanced oestrogen-receptor-positive breast cancer. Lancet 389(10087), 2403-2414 (2016).

4. Harbeck N, Penault-Llorca F, Cortes J et al. Breast cancer. Nat. Rev. Dis. Primers 5(1), 66 (2019).

5. Cardoso F, Harbeck N, Fallowfield L, Kyriakides S, Senkus E. ESMO Guidelines Working Group. Locally recurrent or metastatic breast cancer: ESMO Clinical Practice guidelines for diagnosis, treatment and follow-up. Ann. Oncol. 23(Suppl. 7), vii11-19 (2012).

-• The European Society for Medical Oncology Clinical Practice guidelines describe diagnostic procedures, treatment options and follow-up recommendations for patients with locally recurrent or metastatic breast cancer.

6. Bergh J, Jonsson PE, Lidbrink EK et al. FACT: an open-label randomized phase III study of fulvestrant and anastrozole in combination compared with anastrozole alone as first-line therapy for patients with receptor-positive postmenopausal breast cancer. J. Clin. Oncol. 30(16), 1919-1925 (2012).

7. Mehta RS, Barlow WE, Albain KS et al. Combination anastrozole and fulvestrant in metastatic breast cancer. N. Engl. J. Med. 367(5), 435-444 (2012).

8. Robertson JFR, Bondarenko IM, Trishkina E et al. Fulvestrant $500 \mathrm{mg}$ versus anastrozole $1 \mathrm{mg}$ for hormone receptor-positive advanced breast cancer (FALCON): an international, randomised, double-blind, phase 3 trial. Lancet 388(10063), 2997-3005 (2016).

9. Gradishar W, Salerno KE. NCCN guidelines update: breast cancer. J. Natl. Compr. Canc. Netw. 14(Suppl. 5), 641-644 (2016).

- Summarizes updates to the 2016 National Comprehensive Cancer Network's Clinical Practice guidelines for Breast Cancer, including the addition of palbociclib as a recommended treatment in combination with endocrine therapy for patients with advanced breast cancer.

10. Baselga J, Campone M, Piccart M et al. Everolimus in postmenopausal hormone-receptor-positive advanced breast cancer. $N$. Engl. J. Med. 366(6), 520-529 (2012).

11. Klijn JG, Beex LV, Mauriac L et al. Combined treatment with buserelin and tamoxifen in premenopausal metastatic breast cancer: a randomized study. J. Natl. Cancer Inst. 92(11), 903-911 (2000).

12. Di Leo A, Jerusalem G, Petruzelka L et al. Results of the CONFIRM phase III trial comparing fulvestrant $250 \mathrm{mg}$ with fulvestrant $500 \mathrm{mg}$ in postmenopausal women with estrogen receptor-positive advanced breast cancer. J. Clin. Oncol. 28(30), 4594-4600 (2010). 
13. Di Leo A, Jerusalem G, Petruzelka L et al. Final overall survival: fulvestrant $500 \mathrm{mg}$ vs $250 \mathrm{mg}$ in the randomized CONFIRM trial. J. Natl. Cancer Inst. 106(1), djt337 (2014).

14. Finn RS, Crown JP, Lang I et al. The cyclin-dependent kinase 4/6 inhibitor palbociclib in combination with letrozole versus letrozole alone as first-line treatment of oestrogen receptor-positive, HER2-negative advanced breast cancer (PALOMA-1/TRIO-18): a randomised phase 2 study. Lancet Oncol. 16(1), 25-35 (2015).

- Findings from the PALOMA-1 Phase II clinical study demonstrate the efficacy and safety of palbociclib plus letrozole compared with letrozole alone in patients with estrogen receptor-positive/HER2-negative advanced breast cancer.

15. Rugo HS, Finn RS, Dieras V et al. Palbociclib plus letrozole as first-line therapy in estrogen receptor-positive/human epidermal growth factor receptor 2-negative advanced breast cancer with extended follow-up. Breast Cancer Res. Treat. 174(3), 719-729 (2019).

16. Cardoso F, Senkus E, Costa A et al. 4th ESO-ESMO international consensus guidelines for advanced breast cancer (ABC 4). Ann. Oncol. 29(8), 1634-1657 (2018).

17. Miller TW, Balko JM, Fox EM et al. ER $\alpha$-dependent E2F transcription can mediate resistance to estrogen deprivation in human breast cancer. Cancer Discov. 1(4), 338-351 (2011).

18. Thangavel C, Dean JL, Ertel A et al. Therapeutically activating RB: reestablishing cell cycle control in endocrine therapy-resistant breast cancer. Endocr. Relat. Cancer 18(3), 333-345 (2011).

19. Razavi P, Chang MT, Xu G et al. The genomic landscape of endocrine-resistant advanced breast cancers. Cancer Cell 34(3), 427-438 e426 (2018).

20. Hong SP, Chan TE, Lombardo Y et al. Single-cell transcriptomics reveals multi-step adaptations to endocrine therapy. Nat. Commun. 10(1), 3840 (2019).

21. Toogood PL, Harvey PJ, Repine JT et al. Discovery of a potent and selective inhibitor of cyclin-dependent kinase 4/6. J. Med. Chem. 48(7), 2388-2406 (2005).

22. Cristofanilli M, Turner NC, Bondarenko I et al. Fulvestrant plus palbociclib versus fulvestrant plus placebo for treatment of hormone-receptor-positive, HER2-negative metastatic breast cancer that progressed on previous endocrine therapy (PALOMA-3): final analysis of the multicentre, double-blind, phase 3 randomised controlled trial. Lancet Oncol. 17(4), 425-439 (2016).

-. The Phase III PALOMA-3 clinical study demonstrates the efficacy and safety of palbociclib plus fulvestrant compared with placebo plus fulvestrant in patients with hormone receptor-positive/HER2-negative metastatic breast cancer who had progressed after previous endocrine therapy.

23. Turner NC, Slamon DJ, Ro J et al. Overall survival with palbociclib and fulvestrant in advanced breast cancer. N. Engl. J. Med. 379(20), 1926-1936 (2018).

-• Findings from a prespecified analysis of overall survival data from the PALOMA-3 pivotal clinical study. Palbociclib plus fulvestrant treatment was associated with a longer overall survival than placebo plus fulvestrant, but the results did not meet the prespecified threshold for statistical significance.

24. Bartlett $\mathrm{CH}$, Mardekian J, Cotter MJ et al. Concordance of real-world versus conventional progression-free survival from a phase 3 trial of endocrine therapy as first-line treatment for metastatic breast cancer. PLoS ONE 15(4), e0227256 (2020).

25. Park YH, Kim TY, Kim GM et al. Palbociclib plus exemestane with gonadotropin-releasing hormone agonist versus capecitabine in premenopausal women with hormone receptor-positive, HER2-negative metastatic breast cancer (KCSG-BR15-10): a multicentre, open-label, randomised, phase 2 trial. Lancet Oncol. 20(12), 1750-1759 (2019).

26. Hortobagyi GN, Stemmer SM, Burris HA et al. Updated results from MONALEESA-2, a phase III trial of first-line ribociclib plus letrozole versus placebo plus letrozole in hormone receptor-positive, HER2-negative advanced breast cancer. Ann. Oncol. 29(7), 1541-1547 (2018).

27. Slamon DJ, Neven P, Chia S et al. Phase III randomized study of ribociclib and fulvestrant in hormone receptor-positive, human epidermal growth factor receptor 2-negative advanced breast cancer: MONALEESA-3. J. Clin. Oncol. 36(24), 2465-2472 (2018).

28. Tripathy D, Im SA, Colleoni M et al. Ribociclib plus endocrine therapy for premenopausal women with hormone-receptor-positive, advanced breast cancer (MONALEESA-7): a randomised phase 3 trial. Lancet Oncol. 19(7), 904-915 (2018).

29. Sledge GW Jr, Toi M, Neven P et al. MONARCH 2: abemaciclib in combination with fulvestrant in women with HR+/HER2advanced breast cancer who had progressed while receiving endocrine therapy. J. Clin. Oncol. 35(25), 2875-2884 (2017).

30. Johnston S, Martin M, Di Leo A et al. MONARCH 3 final PFS: a randomized study of abemaciclib as initial therapy for advanced breast cancer. NPJ Breast Cancer 5, 5 (2019).

31. Turner NC, Liu Y, Zhu Z et al. Cyclin E1 expression and palbociclib efficacy in previously treated hormone receptor-positive metastatic breast cancer. J. Clin. Oncol. 37(14), 1169-1178 (2019).

32. Finn RS, Liu $\mathrm{Y}, \mathrm{Zhu} \mathrm{Z}$ et al. Biomarker analyses of response to cyclin-dependent kinase $4 / 6$ inhibition and endocrine therapy in women with treatment-naive metastatic breast cancer. Clin. Cancer Res. 26(1), 110-121 (2020).

33. Tripathy D, Rugo HS, Kaufman PA et al. The SystHERs registry: an observational cohort study of treatment patterns and outcomes in patients with human epidermal growth factor receptor 2-positive metastatic breast cancer. BMC Cancer 14, 307 (2014). 
34. Elson SL, Hiatt RA, Anton-Culver H et al. The Athena Breast Health Network: developing a rapid learning system in breast cancer prevention, screening, treatment and care. Breast Cancer Res. Treat. 140(2), 417-425 (2013).

35. Brufsky AM, Mayer M, Rugo HS et al. Central nervous system metastases in patients with HER2-positive metastatic breast cancer: incidence, treatment and survival in patients from registHER. Clin. Cancer Res. 17(14), 4834-4843 (2011).

36. Kaufman PA, Brufsky AM, Mayer M et al. Treatment patterns and clinical outcomes in elderly patients with HER2-positive metastatic breast cancer from the registHER observational study. Breast Cancer Res. Treat. 135(3), 875-883 (2012).

37. Tripathy D, Kaufman PA, Brufsky AM et al. First-line treatment patterns and clinical outcomes in patients with HER2-positive and hormone receptor-positive metastatic breast cancer from registHER. Oncologist 18(5), 501-510 (2013).

38. Yardley DA, Tripathy D, Brufsky AM et al. Long-term survivor characteristics in HER2-positive metastatic breast cancer from registHER. Br. J. Cancer 110(11), 2756-2764 (2014).

39. Syrios J, Pappa E, Volakakis N et al. Real-world data on health-related quality of life assessment in patients with breast cancer receiving subcutaneous trastuzumab. Breast Cancer (Auckl.) doi: 10.1177/1178223418758031 (2018).

40. Booth CM, Karim S, Mackillop WJ. Real-world data: towards achieving the achievable in cancer care. Nat. Rev. Clin. Oncol. 16(5), 312-325 (2019).

41. Fayers PM, Aaronson NK, Bjordal K et al. EORTC QLQ-C30 Scoring Manual. European Organisation for Research and Treatment of Cancer, Brussels, Belgium (2001).

42. Wallwiener M, Matthies L, Simoes E et al. Reliability of an e-PRO tool of EORTC QLQ-C30 for measurement of health-related quality of life in patients with breast cancer: prospective randomized trial. J. Med. Internet Res. 19(9), e322 (2017).

43. Dottorini L, Catena L, Sarno I et al. The role of Geriatric Screening Tool (G8) in predicting side effect in older patients during therapy with aromatase inhibitor. J. Geriatr. Oncol. 10(2), 356-358 (2019).

44. DeSanto-Madeya S, Bauer-Wu S, Gross A. Activities of daily living in women with advanced breast cancer. Oncol. Nurs. Forum 34(4), 841-846 (2007).

45. Nolte S, Liegl G, Petersen MA et al. General population normative data for the EORTC QLQ-C30 health-related quality of life questionnaire based on 15,386 persons across 13 European countries, Canada and the United States. Eur. J. Cancer 107, 153-163 (2019).

46. Hurria A, Levit LA, Dale W et al. Improving the evidence base for treating older adults with cancer: American Society of Clinical Oncology statement. J. Clin. Oncol. 33(32), 3826-3833 (2015).

- Recommendations from the American Society of Clinical Oncology to increase the quantity and quality of research evaluating treatments for older adults with cancer, including leveraging the eligibility criteria and study design of clinical studies and requirements or incentives for studies to include older adults.

47. Hutchins LF, Unger JM, Crowley JJ, Coltman CA Jr, Albain KS. Underrepresentation of patients 65 years of age or older in cancer-treatment trials. N. Engl. J. Med. 341(27), 2061-2067 (1999).

48. Kornblith AB, Kemeny M, Peterson BL et al. Survey of oncologists' perceptions of barriers to accrual of older patients with breast carcinoma to clinical trials. Cancer 95(5), 989-996 (2002).

49. Shachar SS, Hurria A, Muss HB. Breast cancer in women older than 80 years. J. Oncol. Pract. 12(2), 123-132 (2016).

50. Strulov Shachar S, Hurria A, Muss HB. Targeted therapies in older adults with breast cancer: what do we know? J. Clin. Oncol. 34(28), 3486-3488 (2016).

51. Levit LA, Singh H, Klepin HD, Hurria A. Expanding the evidence base in geriatric oncology: action items from an FDA-ASCO workshop. J. Natl Cancer Inst. 110(11), 1163-1170 (2018).

52. Imran M, Al-Wassia R, Alkhayyat SS, Baig M, Al-Saati BA. Assessment of quality of life (QoL) in breast cancer patients by using EORTC QLQ-C30 and BR-23 questionnaires: a tertiary care center survey in the western region of Saudi Arabia. PLoS ONE 14(7), e0219093 (2019)

53. Wedding U, Pientka L, Hoffken K. Quality-of-life in elderly patients with cancer: a short review. Eur. J. Cancer 43(15), 2203-2210 (2007).

54. Wöckel A, Fasching PA, Guderian G et al. RIBANNA - real-world evidence of ribociclib plus aromatase inhibitor, or endocrine monotherapy, or chemotherapy as first-line therapy for postmenopausal women with HR+, HER2- advanced breast cancer (aBC). Cancer Res. 79(Suppl. 4), Abstract nr OT3-02-01 (2019). 